\title{
An update in the management of spinal metastases
}

\author{
Atualização no manejo das metástases na coluna vertebral \\ Andrei F. Joaquim', Ann Powers², Ilya Laufer², Mark H. Bilsky²
}

\begin{abstract}
The best clinical treatment for spinal metastases requires an integrated approach with input from an interdisciplinary cancer team. The principle goals of treatment are maintenance or improvement in neurologic function and ambulation, spinal stability, durable tumor control, and pain relief. The past decade has witnessed an explosion of new technologies that have impacted our ability to reach these goals, such as separation surgery and minimally invasive spinal procedures. The biggest advance, however, has been the evolution of stereotactic radiosurgery that has demonstrated durable tumor control both when delivered as definitive therapy and as a postoperative adjuvant even for tumors considered markedly resistant to conventional external beam radiation. In this paper, we perform an update on the management of spinal metastases demonstrating the integration of these new technologies into a decision framework NOMS that assesses four basic aspects of a patient's spine disease: Neurologic, Oncologic, Mechanical Instability and Systemic disease.
\end{abstract}

Keywords: spinal metastases, bone metastases, diagnosis, management, treatment, NOMS.

\section{RESUMO}

O tratamento dos pacientes com metástases na coluna requer uma abordagem multidisciplinar por equipe especializada em oncologia. Os objetivos básicos do tratamento são a manutenção/ melhora da função neurológica com preservação da deambulação, manutenção da estabilidade da coluna, controle tumoral e alívio da dor. A última década testemunhou uma explosão de novas tecnologias que auxiliaram a atingir os objetivos terapêuticos, como a cirurgia de separação e procedimentos minimamente cirúrgicos minimamente invasivos. Contudo, o maior avanço terapêutico constitui-se do uso da radiocirurgia no tratamento das metástases de coluna, que possibilita bom controle local tanto como terapia definitiva ou no pós-operatório de tumores, mesmo os considerados radioresistentes à radioterapia convencional. No presente artigo, realizamos atualização do manejo das metástases de coluna, apresentando a integração das novas tecnologias em um algoritmo de decisão "NOMS" que inclui os quatros aspectos básicos dos pacientes com metástases na coluna: Neurologic, Oncologic, Mechanical Instability e Systemic disease.

Palavras-chave: metastases de coluna, metástases ósseas, diagnóstico, manejo, tratamento, NOMS.

Advances in cancer treatment over the past decade, such biologics and immunotherapy are improving patient outcomes and consequently life expectancy. The most common sites for metastases in the general population with cancer are the liver and lungs, followed by bone. Considering bone metastases, the majority will affect the spine ${ }^{1}$. Some studies estimate that up to $40 \%$ of all patients with cancer will have spinal metastases (SM) during the course of their disease $e^{2,3}$.

The most frequent histologic types of cancer that give rise to bone metastases are breast, prostate and lung cancer $^{4}$. Most of the spinal metastases are diagnosed following the diagnosis of the primary cancer. However, in about $10 \%$ of the patients, SM is the first manifestation of an unknown primary tumor ${ }^{1}$.

Spinal cord compression is expected in up to $20 \%$ of the patients with SM. Approximately $95 \%$ of the patients with SM will demonstrate epidural metastases, mainly affecting the vertebral body and the pedicle regions, whereas $5 \%$ will present with intradural and less than $1 \%$ with intramedullary metastases ${ }^{5,6}$. Symptomatic spinal cord compression occurs more frequently in the thoracic spine, followed by cervical and then lumbar. The greater incidence of SM in the thoracic spine is attributed to the higher number of vertebrae and small canal diameter?

'Universidade Estadual de Campinas, Departamento de Neurologia, Divisão de Neurocirurgia, Campinas SP, Brazil;

${ }^{2}$ Memorial Sloan Kettering Cancer Center, Department of Neurosurgery, New York NY, USA.

Correspondence: Andrei F. Joaquim; UNICAMP, Disciplina de Neurocirurgia; Rua Antonio Lapa, 280/sala 506; $13025-240$ Campinas SP, Brasil;

E-mail: andjoaquim@yahoo.com

Conflict of interes: There is no conflict of interest to declare.

Received 05 January 2015; Received in final form 15 April 2015; Accepted 04 May 2015. 
The optimal clinical management of these patients requires integrated decisions by an interdisciplinary cancer team comprised of medical and radiation oncologists and spine surgeons, as well as all other health care professionals (e.g. rehabilitation team, nurses, and other involved medical specialties) $)^{8,9}$. Survival is based on the primary tumor histology, systemic disease status, and also patient comorbidities, resulting in a very heterogeneous group ${ }^{8}$. Of note, only 10 to $20 \%$ of the patients with SM will be alive after two years after the diagnosis, which must be taken into account when considering a patient's treatment ${ }^{8,9}$. With the introduction of newer biologics and immunotherapy for tumors such as renal cell carcinoma and melanoma, overall survival is improving leading to need to provide a more durable solution for spine metastases. Considering the life expectancy, treatment of patients with SM is palliative with the aim of achieving five principle goals: 1) pain control, 2) improvement in quality of life, 3) spine stabilization, 4) maintenance or improvement of neurological status, and 5) local disease control ${ }^{8}$.

New treatment modalities have improved the treatment of spine metastases over the past decade. Surgical advances include less invasive approaches, such as separation surgery, pedicle screw fixation which can be placed via either an open or percutaneous approach and cement augmentation techniques ${ }^{9,10}$. However, by far, the greatest impact has been the evolution and integration of spine stereotactic radiosurgery (SRS), typically delivered as 16 to 24 Gy single fraction or 24 to 30 Gy in 3 fractions 9 The ability to deliver high-dose conformal radiation in ablative doses has improved local control rates from $30 \%$ at 3 months using conventional external beam radiotherapy (cEBRT) (e.g. $30 \mathrm{~Gy}$ in 10 fractions) to over $90 \%$ when used as definitive therapy or as postoperative adjuvant ${ }^{11,12,13,14,15}$.

In this review, we perform an update based on the NOMS decision framework of the concepts involved in the management of SM, considering the basic assessments necessary to choose the best treatment modality for an individual patient.

\section{PATIENT'S EVALUATION - TREATMENT DECISION PROCESS}

Many scoring system have been proposed to estimate the prognosis of patients with SM, an important factor in the decision of treatments to be performed. The modified Tokuhashi Scoring System is a prognostic scale that evaluates patients Karnofsky score, neurological status, number of bone metastases outside spine, metastasis to major organs and the primary tumor site ${ }^{16}$. The system is used to estimate survival, which is very important in the decision-making. Another popular scoring system developed to aid in predicting survival is the Tomita score that considers the primary tumor site, visceral metastases and bone metastases ${ }^{17}$. Both systems were proposed for helping in the choice of palliative versus aggressive surgery based on estimated patient survival. However, these algorithms have been criticized because they do not consider the presence of instability, or the significant impact of advances in radiation therapy, such as radiosurgery. Further, these algorithms have not reassessed the extended survivals demonstrated in tumors such as renal cell carcinoma and melanoma with the integration of newer biologics and immunotherapy ${ }^{18}$.

Rather than using algorithms that are fixed in time and constrained by technology available at the time they were proposed, a new decision framework NOMS has been developed that considers four sentinel decision points in decision making. ${ }^{8}$. NOMS consists of four fundamental assessments: Neurologic, Oncologic, Mechanical Instabilty, and Sytemic Disease $^{8}$. Unlike the Tokuhashi and Tomita algorithms, the advantage of the NOMS framework is that it incorporates both new technologies and evidenced based medicine as they become available. Considering these four assessments, the interdisciplinary team can determine the optimal treatment consisting of radiation therapy (Table 1), surgery, systemic therapy, or a combination of these. In the NOMS decision framework, the neurologic assessment principally reflects the degree of epidural spinal cord compression (ESCC) as well as the presence or absence of myelopathy and/or functional radiculopathy ${ }^{8}$. Spinal cord compression is based on a validated scoring system using magnetic resonance (MR) axial T2-weighed images ${ }^{8}$. This scoring system is used to differentiate no or minimal ESCC (0-1c) from high-grade spinal ESCC 2-3 (Table 2) (1) $^{8}$. The oncologic consideration is predicated on the known cytotoxicity and the durability of the response to current treatment modalities including cEBRT, SRS, chemotherapy, hormones, immunotherapy, or biologics. In terms of malignant tumors, surgery plays a very limited role in tumor control. Mechanical instability has recently been defined for neoplastic disease and a scoring system, spinal instability neoplastic score (SINS), has been developed to aid in this assessment ${ }^{20}$. The recognition of spinal instability resulting from tumor is imperative because an unstable spine will not respond to radiation and/or chemotherapy, but requires an intervention, such as brace application, percutaneous cement augmentation and/or pedicle screws, or open surgery. The final assessment in NOMS reflects the extent of systemic disease, medical co-morbidities and expected survival, which all impact the decision to offer not only surgical treatment, but also radiation or systemic therapy.

The neurologic and oncologic assessments are considered in combination ${ }^{8}$. From the oncologic perspective, radiation is the mainstay of therapy for tumor control. With few exceptions, such as myeloma and lymphoma, systemic therapy has little impact in this regard. It is unknown whether the newer biologics, such as sorafenib for renal cell carcinoma, have the same responses in bone metastases as visceral disease even if the driver mutation is present ${ }^{21}$. Our experience with the use of biologics in treating SM is somewhat mixed and inconsistent, and on this basis, is not considered in the 
Table 1. Current NOMS decision framework.

\begin{tabular}{|c|c|c|c|c|}
\hline Neurologic & Oncologic & Mechanical & Systemic & Decision \\
\hline Low Grade ESCC / No Myelopathy & Radiosensitive & Stable & --- & cEBRT \\
\hline Low Grade ESCC / No Myelopathy & Radiosensitive & Unstable & --- & Stabilization* followed by cEBRT \\
\hline High Grade ESCC +/- Myelopathy & Radiosensitive & Stable & --- & cEBRT \\
\hline High Grade ESCC +/- Myelopathy & Radiosensitive & Unstable & --- & Stabilization* followed by cEBRT \\
\hline Low Grade ESCC / No Myelopathy & Radioresistant & Stable & --- & IGRT \\
\hline Low Grade ESCC / No Myelopathy & Radioresistant & Unstable & --- & Stabilization* followed by IGRT \\
\hline High Grade ESCC +/- Myelopathy & Radioresistant & Stable & Able to tolerate surgery & Decompression/Stabilization followed by IGRT \\
\hline High Grade ESCC +/- Myelopathy & Radioresistant & Stable & Unable to tolerate surgery & cEBRT \\
\hline High Grade ESCC +/- Myelopathy & Radioresistant & Unstable & Able to tolerate surgery & Decompression/Stabilization followed by IGRT \\
\hline High Grade ESCC +/- Myelopathy & Radioresistant & Unstable & Unable to tolerate surgery & Stabilization* followed by cEBRT \\
\hline \multicolumn{5}{|c|}{$\begin{array}{l}\text { ESCC Scale } 0 \text { and } 1 \text { = Low Grade ESCC; ESCC Scale } 2 \text { and } 3=\text { High Grade ESCC; } * \text { Stabilization options include percutaneous cement augmentation } \\
\text { percutaneous pedicle screw instrumentation and open instrumentation. In patients with significant systemic comorbidities limiting their ability to tolerate } \\
\text { open surgery, stabilization may be limited to cement augmentation and / or percutaneous screw augmentation. } \\
\text { NOMS: neurologic, oncologic, mechanical instability and systemic disease; ESCC: epidural spinal cord compression; cEBRT: conventional External Beam } \\
\text { Radiotherapy; IGRT image-guided radiation therapy. }\end{array}$} \\
\hline
\end{tabular}

setting of high-grade spinal cord compression or symptomatic lesions. The two modalities of radiation currently available for the treatment of spine metastases are conventional external beam radiation (cEBRT - e.g. 30 Gy in 10 fractions) and stereotactic radiosurgery (SRS - e.g. 16 to 24 Gy single fraction or 24 to 30 Gy in 3 to 5 fractions) $)^{10,11,12,13,14,15,22}$. These hypofractionated radiation doses are ablative to the tumor while sparing normal tissue tolerance. Mounting evidence suggests that the radiobiology of SRS is different from conventionally fractionated radiation ${ }^{23}$. In combination with the oncologic considerations, the neurologic assessment is one of the critical determinants in deciding between these modalities as cEBRT can be used in the setting of high-grade ESCC (ESCC 0 to 3), but the safe delivery of SRS requires a 2 to $3 \mathrm{~mm}$ margin on the spinal cord in order to avoid radiation myelitis ${ }^{24}$. Although groups are studying the use of SRS in the setting of high-grade spinal cord compression, most commonly SRS use is restricted to tumors confined to the bone or with minimal epidural impingement (ESCC 0-1c) ${ }^{8,24}$.

\section{Radiosensitive tumors: Conventional External Beam Radiation}

Patients with radiosensitive tumors can be treated effectively with cEBRT regardless of the degree of $\mathrm{ESCC}^{8}$. A review of the literature shows that tumor histology is perhaps the most important factor in determining response to cEBRT ${ }^{9}$. Among patients who underwent cEBRT in the setting of spinal metastases, the mean ambulation rate was $81 \%$ (range from $58 \%$ to $100 \%)^{9}$. However, only $6 \%$ to $67 \%$ percent of non-ambulatory patients' recovered ambulation, with reports in the higher range thought to be attributable to the large number of favorable histologies in those series ${ }^{11,13}$. Literature analysis reveals that all authors classify lymphoma, seminoma, and myeloma as radiosensitive histologies and supports the use of cEBRT to treat these tumors, regardless of the degree of ESCC or neurologic deficit ${ }^{11,13,25,26,27}$. On the other hand, solid tumors exhibit a wide range of radiosensitivity ${ }^{25-29}$. Radiosensitive solid tumor histologies include breast, prostate, ovarian, and
Table 2. Epidural spinal cord compression scoring system based on MR axial T2-weighted images.

0 : tumor confined to bone,

1: tumor extension into the epidural space without deformation of the spinal cord,

1a: epidural impingement but no deformation of the thecal sac;

$1 \mathrm{~b}$ : deformation of the thecal sac but without spinal cord abutment;

1c: deformation of the thecal sac with spinal cord abutment but without compression

2: spinal cord compression but CSF is visible and

3: spinal cord compression without visible CSF

MR: magnetic resonance; CSF: cerebrospinal fluid.

neuroendocrine carcinomas. Renal, thyroid, hepatocellular, colon, and non-small cell lung carcinomas, sarcoma, and melanoma represent radioresistant tumors ${ }^{25,26,27,28,29}$. Solid tumors with radioresistant histologies generally require SRS to achieve durable local control, whereas radiosensitive solid tumors may be treated with cEBRT or SRS ${ }^{15,25,26,27,28,29}$.

\section{Radioresistant Tumor without spinal cord compression (ESCC 0 to 1c): SRS}

Unlike the poor responses to cEBRT, responses to SRS now demonstrate greater than $90 \%$ durable response rates when used as definitive treatment in patients with minimal or no spinal cord compression (ESCC 0 to 1c). In a review of 413 patient undergoing single fraction SRS with doses ranging from 18 to 24 Gy, Yamada, et al., reported 3-year recurrence rates of $4 \%$ when censored for death that were histology independent with a dose response demonstrating that those who received greater $24 \mathrm{~Gy}$ had a better local tumor control than those who received less than $24 \mathrm{~Gy}^{12}$. Guckenberger et al. reported a multi-institutional retrospective review of $301 \mathrm{pa}-$ tients with 387 vertebral body metastases ${ }^{29}$. The median treatment dose was $24 \mathrm{~Gy}$ in 3 fractions with 2-year local control rates reported at $83.9 \%$. In this study, tumor histology (i.e. non-small lung carcinoma, melanoma, and renal cell carcinoma) were associated with worse outcomes for local tumor 
control $^{29}$. The application of SRS has transformed tumors historically considered resistant to cEBRT into very responsive tumors. The responses to SRS are histology independent unlike those seen with cEBRT.

\section{Radioresistant Tumors with High-grade spinal cord compression (ESCC 2 to 3 ): Surgery + SRS}

From the neurologic and oncologic perspective, surgery is reserved for patients with radioresistant tumors who have high-grade spinal cord compression. Surgery is used to preserve or restore neurologic function and stabilize the spine. The justification for surgery in patients with high-grade spinal cord compression is primarily based on a prospective randomized trial published by Patchell et al. comparing surgery followed by cEBRT to cEBRT alone ${ }^{30}$. Statistically significant improvements were found in the surgical group with regard to maintenance and recovery of ambulation, bowel and bladder continence, narcotic requirements, and survival. Despite limitations, this study was sentinel in establishing that neurological outcomes were significantly better in treating myelopathic patients with radioresistant tumors. Radiosensitive tumors, such as lymphoma and myeloma, were excluded as this would have heavily biased the study toward the radiation arm and would not have answered the principal question regarding the best treatment for solid tumor malignancies. At the time the study was designed, instability was not recognized as a contraindication to radiation as a primary treatment. Without recognizing this concept, $30 \%$ in each arm were classified as unstable by the Cybulski criteria, which biased the outcome toward the surgical arm. Additionally, the duration of compression was longer in the irradiated arm potentially biasing the improved outcomes toward surgery. The definition of ambulation was also fairly limited, i.e., the ability to walk two steps: however, using this definition, the differences in both maintaining and recovering ambulation were significant.

Finally, despite surgery providing neurologic salvage, durable tumor control remained a problem when using cEBRT as a postoperative adjuvant with recurrence rates of approximately $70 \%$ at one-year follow-up. A major risk factor for recurrence is radioresistant-tumor histology ${ }^{8}$. The transition to using of SRS as a postoperative adjuvant has substantially improved local control rates compared to cEBRT while reducing the need for aggressive surgical approaches, such as en bloc spondylectomy.

\section{M - Mechanical instability}

Mechanical instability is a separate assessment from the neurologic and oncologic evaluation as no amount of radiation will stabilize an unstable spine. Mechanical instability in the setting of neoplastic spinal disease can be evaluated used the SINS (Table 3), a new comprehensive classification system recently proposed that is based on patient symptoms and radiographic criteria in an attempt to predict eventual spinal instability ${ }^{20}$.
The SINS includes the following assessments: 1) tumor location, 2) type and presence of pain, 3) bone lesion quality, 4) spinal alignment, 5) extent of vertebral body collapse and 6) posterolateral elements involvement. A qualitative score is them obtained, and instability is suggested according to the final score. The minimum score is 0 and maximum is 18 . Stability is denoted when the score is 0 to 6 ; indeterminate instability is proposed when the score of 7 to 12 and frank instability is determined when the injury has a score of 13 to 18 . Surgical consultation is recommended when patients had a score of 7 to 18. Mechanical instability is an indication for surgical stabilization, regardless of tumor radioresistance to cEBRT or histology ${ }^{20}$.

\section{S - Systemic disease}

Systemic disease and medical comorbidities evaluate the ability of the patient to tolerate a proposed treatment and can influence patient's survival as well as the oncological status. The oncology team can help in stratify surgical risk according to tumor histology. Some tumors have median survival of less than 6 months, such as non-small cell lung carcinoma ${ }^{10}$. In such situations, surgical treatment can be not advisable. The same is true, for instance, in the setting of a severe heart ischemic disease or chronic pulmonary obstructive disease,

Table 3. The SINS - Spinal Instability Neoplastic Score Element of SINS Score.

Location
Junctional (occiput-C2, C7-T2, T11-L1, L5-S1)
Mobile spine (C3-C6, L2-L4)
Semi-rigid (T3-T10)
Rigid (S2-S5)

Pain relief with recumbency and/or pain with movement/ loading of the spine

Yes 3

No (occasional pain but not mechanical) 1

Pain free lesion 0

Bone lesion

Lytic 2

Mixed (lytic/blastic)

Blastic

0

Radiographic spinal alignment

Subluxation/translation present 4

De novo deformity (kyphosis/scoliosis) 2

Normal alignment

Vertebral body collapse

$>50 \%$ collapse 3

$<50 \%$ collapse $\quad 2$

No collapse with > 50\% body involved 1

None of the above 0

Posterolateral involvement of the spinal elements (facet, pedicle or CV joint fracture or replacement with tumor)

Bilate ral 3

Unilateral 1

None of the above 0

Score 0-6: No surgical consultation is required; Score 7-18: Surgical consultation is advisable. 
where a surgical procedure can be prohibitive. In summary, the extent of systemic disease and functional status as assessed by patient comorbidities can preclude surgical treatment in selected cases.

\section{DIAGNOSTIC WORK-UP}

Basic routine tests of patients with SM include: complete blood count with differential, coagulation test, renal function assessment, electrolytes, among others. Specific evaluation is guided according to clinical presentation and tumor characteristics and primary site. Radiological assessment of systemic metastases is critical except in cases where urgent treatment is required, such as a progressive acute deficit.

Although plain radiographies and CT scanning can be useful for surgical planning and to evaluate spinal deformities, MR is the imaging modality of choice for SM evaluation ${ }^{30}$. Typically sagittal screening of the entire spinal axis is undertaken to assess for occult lesions outside the symptomatic area that may bear on decision making. The most important sequences for sagittal screening are T1-weighted and STIR images in which tumor is hypointense and hyperintense, respectively. Specific MRI sequences can help radiologists to differentiate degenerative changes and osteoporotic fractures from metastases ${ }^{8,31}$. As previously noted, the degree of spinal cord compression is predicated on axial T2-weighted sequences. Most commonly images are obtained with and without gadolinium contrast to further evaluate the degree of spinal cord compression and to rule out leptomeningeal and intramedullary tumors. More recently perfusion images are being explored to assess the viability of tumors. Dynamic contrast-enhanced MR perfusion images particularly evaluating plasma volume are very sensitive and specific for assessing treatment responses following SRS even before changes are seen on standard MR imaging ${ }^{32}$.

Positron Emission Tomography (PET-CT) is also useful imaging modalities for screening systemic disease and other metastases that can influence the treatment planned PET has often been used to differentiate pathologic from osteoporotic or traumatic spine fractures. Laufer et al. reviewed 82 patients who had needle biopsy of a suspicious lesion within 6 week of a fluorodeoxyglucose (FDG) PET scan ${ }^{33}$. The mean standardized uptake value (SUV) for tumor was 7.1 versus 2.1 for benign lesions. In patients with lytic or mixed lytic/blastic from solid tumor metastases, there was a $100 \%$ concordance between FDG-PET and needle biopsy when using an SUV cutoff of $2^{33}$.

\section{MEDICAL MANAGEMENT}

Considering medication, treatment of pain is of paramount importance. Pain is the most common clinical manifestation of $\mathrm{SM}^{31}$. Metastatic spine patients typically present with either biologic or instability pain. Biologic pain is night or morning pain that resolves over the course of the day. The suspected pathophysiology is the diurnal variation in endogenous steroid secretion which decreases during sleep. The flare pain results from inflammatory mediators secreted by the tumor. Identifying the cause of pain is important to guide proper treatment: biologic pain often responds to steroids and radiation, but pain secondary to spinal instability may require a surgical procedure for stabilization. Medication for SM included non-steroidal anti-inflammatories and steroids. Steroids can be also used in the setting of neurological impairment due to tumor compression: generally, a loading $10 \mathrm{mg}$ dose of dexamethasone followed by $4 \mathrm{mg}$ every 6 hours can decrease neurological symptoms secondary to compression as well as local pain 7 . However, doses should be decreased as soon as possible to avoid side effects such as cognitive dysfunction, gastrointestinal bleeding and glucose intolerance ${ }^{31}$. Patients on long-term steroids are placed on trimethoprim/sulfamethoxazole three times per week as pneumocystic prophylaxis. Opioids are also important medication to control pain, especially because the risk of addiction is not a major concern in cancer patients. Some patients also may require antiepileptic drugs for treating neuropathic pain, such as tricyclics and gabapentin. Considering patient's systemic status and general condition is important prior to prescribe any pain medication ${ }^{34}$.

\section{SURGICAL PROCEDURES}

\section{Biopsy}

Biopsy is advisable in all patients without a diagnosis of a primary tumor and for spine tumors that represent the first sign of metastasis ${ }^{35}$. It can be performed via a percutaneous needle biopsy guided by fluoroscopy or CT scan or an open biopsy. Especially in the setting of spinal cord compression, it is important to have a fast tissue analysis to evaluate tumor sensitivity to radiation therapy ${ }^{35}$. In the setting of acute and rapid progressive neurological deficits, waiting for histopatological analysis can result in severe neurological impairment and a surgical procedure for decompression and obtain tissue samples can be preferred than a needle biopsy. Biopsy should also be considered when radiological findings are inconsistent with tumor, requiring differential diagnosis, such as an infection process or in primary bone tumors.

\section{Open surgery}

One obvious but basic principle for surgical treatment is that the patient can tolerate the procedure. Most authors considered that life expectancy should be of more than 3 months to justify a surgical approach. However, patients' with severe pain due to instability or in specific selected cases may have benefits of small surgical procedures for stabilization/ decompression if they can tolerate it, after thorough multidisciplinary analysis ${ }^{9,10}$. 
Instability, evaluated by the SINS, is an indication for surgery that is independent of other variables, such as tumor histology or radiosensitivity ${ }^{8,20}$. Modern spinal instrumentation with anterior and/ or posterior fixation can decrease the pain and improve neurological function secondary to spinal instability ${ }^{10,35}$. Minimally invasive techniques can be used to decrease surgical morbidity. Of note, bone graft is typically used in spine procedures, but the expectation of arthrodesis is low due to the short life and the use of radiation and systemic therapy.

Simple laminectomy without instrumentation is not routinely used because it will potentially create iatrogenic instability of the spine involved by tumor ${ }^{30}$. However, in selected cases of tumor involving only the posterior elements or epidural tumor without bone involvement, laminectomy is a reasonable surgical option. Of note, these patients can require further procedures for spine stabilization, considering the potential risk of development of a deformity resulting from radiation ${ }^{10}$.

Surgery can provide spinal stabilization and spinal cord decompression, but local tumor control is made dependent on effective radiation therapy. cEBRT can achieve local control in more than $80 \%$ of the cases in radiosensitive tumors after 2 years $^{22}$. However, control rates with cEBRT are of less than $50 \%$ after 2 years in radioresistant histologies ${ }^{36}$. Before image-guided techniques for radiation therapy, more aggressive excisions were required for treating radioresistant tumors, which resulted in increased morbidity and extended procedures in these severely ill patients. SRS has demonstrated local control of up to $90 \%$ even in histologies considered poor responsible for $\mathrm{cE}$ BRT, such as melanoma and renal cell carcinoma ${ }^{14,15}$. The need for cytoreductive surgery has decreased over the past 10 years for treating SM with the high tumor control rates obtained using SRS has changed the paradigm of maximal tumor resection to the era of "separation" surgery - a procedure that separates the tumor from the dural sac to allow good conditions for delivery radiation therapy after the procedure ${ }^{10}$.

Separation surgery is used to decompress the spinal cord in order to reconstitute the spinal fluid space in conjunction with a long screw-rod instrumented fusion (Figure $)^{10}$. Tumor in the vertebral body and large paraspinal masses are not resected which reduces the time and morbidity from more extensive surgical approaches and aggressive tumor resection. This decompression provides a safe margin to deliver a cytotoxic radiation dose to the tumor within the constraints of spinal cord tolerance. Stabilization depends on the degree of instability, but usually fixation is performed with pedicle or lateral mass screws two levels above and two levels below the affected level, with cement augmentation in cases where poor bone quality is observed. In this context, anterior reconstruction is rarely required, decreasing blood loss and surgical time significantly ${ }^{10}$. Moulding et al. reported the outcomes using high-dose (18-24 Gy) single fraction SRS after separation surgery resulted in local disease control rates with an estimated 1 -year local failure risk of only $6.3 \%{ }^{24}$. In a review of 186 patients undergoing separation surgery with postoperative SRS, Laufer et al. reported a 1-year estimated cumulative incidence of recurrence of $16.4 \%^{10}$. Patients receiving low-dose hypofractionated radiation (eg.30 Gy in 5 fractions) had local recurrence rate of $22.6 \%$ compared to high-dose hypofractionated (24 to 30 Gy in 3 fractions) or high-dose single fraction (24 Gy) with local recurrence rates of $4.1 \%$ and $9.0 \%$, respectively. Of the tumors treated, 144 (77\%) were considered radioresistant to cEBRT and 91 (49\%) had previously failed cEBRT ${ }^{10}$.

Some authors proposed en bloc resection for metastatic tumors of the spine to avoid local recurrence and to improve long term survival ${ }^{37}$. Potential indications may include a solitary metastatic tumor in the setting of a well controlled primary cancer. Most of the evidence for this aggressive treatment is based on small case series and the morbidity of the
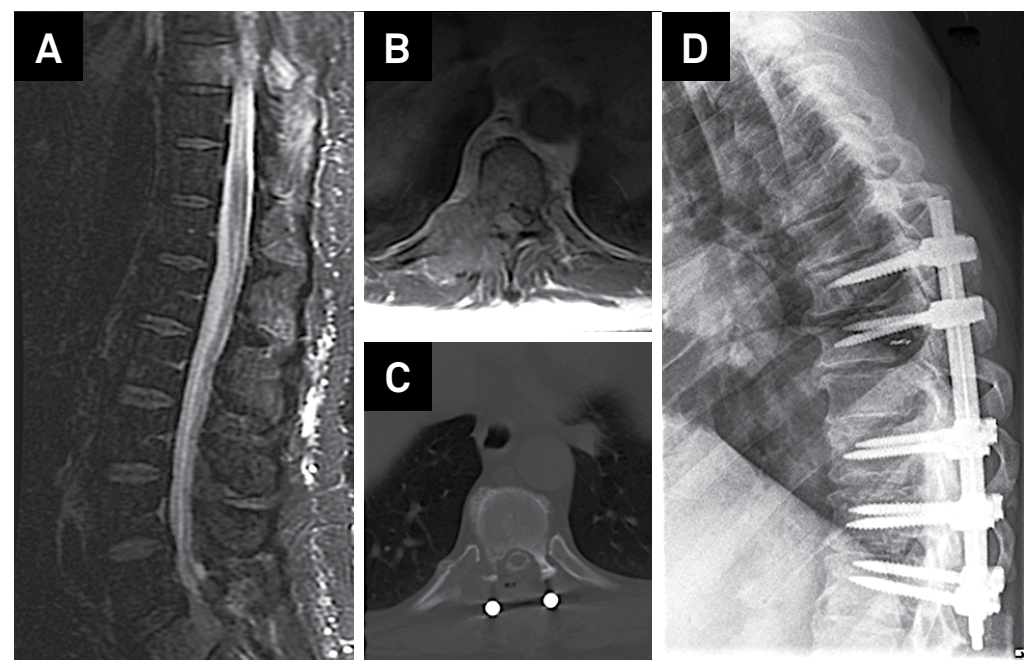

Figure. A and B) A 59 years-old man with renal cell carcinoma metastatic to T9 causing high-grade spinal cord compression. Patient was ambulatory without neurologic deficit. C) Patient underwent separation surgery with T8-10 laminectomy, circumferential spinal cord decompression and D) T7-T12 posterolateral instrumentation and fusion. 
procedure should strongly taken into account the context of an oncologic patient with spinal metastases and a limited life expectancy. Further studies are necessary to demonstrate the value of aggressive surgery compared with the advantages of new treatment modalities in local disease control and long term survival of spinal metastases.

\section{MINIMALLY INVASIVE AND PERCUTANEOUS PROCEDURES}

Advancements in surgical instrumentation resulted in the development of the armamentarium for minimally invasive surgical (MIS) techniques. In an attempt to decrease the morbidity of surgical procedures, allowing faster wound healing and shorter hospital stays, percutaneous augmentation techniques such as vertebroplasty and kyphoplasty as well as percutaneous pedicle screws fixation and decompression may play a role in the treatment of SM. The principle indication is mechanical instability without spinal cord compression. While percutaneous vertebral body cement augmentation is somewhat controversial in the osteoporotic population, Berenson et al. reported the results of a prospective, randomized trial in patients with painful vertebral compression fractures resulting from cancer showing significant improvement in the Roland Morris Disability Questionnaire in patients undergoing kyphoplasty compared to controls ${ }^{38}$.
The integration of percutaneous pedicle screws as an adjunct to vertebral body cement augmentation is principally at the thoracolumbar junction in tumors creating a burst fracture with posterior element involvement. These patients typically need a posterior tension band in addition to anterior support.

The indications for MIS are similar to open surgical, but it is important to recognize surgeons and techniques limitation. For instance, circumferential decompression using MIS can be challenge even in experience hands, as well as approaching vascularized tumors due to difficult in obtain hemostasis.

\section{FINAL REMARKS}

Spinal metastases management require an integrate decision of an interdisciplinary cancer team. The NOMS decision framework has facilitated the choice of the best way to manage these patients. The evaluation of instability due to SM can be performed using the SINS. cEBRT and SRS resulted in high rates of local tumor control in radiosensitive and radioresistant histologies. Separation surgery provides optimal conditions for postoperative radiation therapy and avoids the morbidity of larger excisional surgeries. Minimally invasive surgical techniques, such as percutaneous pedicle screws, endoscopic decompressions and percutaneous vertebral augmentation can decrease surgical morbidity and potentially improves patient's outcomes.

\section{References}

1. Delank KS, Wendtner C, Eich HT, Eysel P. The treatment of spinal metastases. Dtsch Arztebl Int. 2011;108(5):71-9. doi:10.3238/arztebl.2011.007

2. Walsh GL, Gokaslan ZL, McCutcheon IE, Mineo MT, Yasko AW, Swisher SG et al. Anterior approaches to the thoracic spine in patients with cancer: indications and results. Ann Thorac Surg. 1997;64(6):1611-8. doi:10.1016/S0003-4975(97)01034-5

3. Ortiz Gómez JA. The incidence of vertebral body metastases. Int Orthop. 1995;19(5):309-11. doi:10.1007/BF00181116

4. Greenlee RT, Murray T, Bolden S, Wingo PA. Cancer statistics, 2000. CA Cancer J Clin. 2000;50(1):7-33. doi:10.3322/canjclin.50.1.7

5. Perrin RG, Livingston KE, Aarabi B. Intradural extramedullary spinal metastasis: a report of 10 cases. J Neurosurg. 1982;56(6):835-7. doi:10.3171/jns.1982.56.6.0835

6. Schick U, Marquardt G, Lorenz R. Intradural and extradural metastases. Neurosurg Rev. 2001;24(1):1-5. doi:10.1007/PL00011959

7. Bucholtz JD. Metastatic epidural spinal cord compression. Semin Oncol Nurs. 1999;15(3):150-9. doi:10.1016/S0749-2081(99)80002-3

8. Laufer I, Rubin DG, Lis E, Cox BW, Stubblefield MD, Yamada $Y$ et al. The NOMS framework: approach to the treatment of spinal metastatic tumors. Oncologist. 2013;18(6):744-51. doi:10.1634/theoncologist.2012-0293

9. Bilsky MH, Laufer I, Burch S. Shifting paradigms in the treatment of metastatic spine disease. Spine (Phila Pa 1976). 2009;34(22 suppl):S101-7. doi:10.1097/BRS.0b013e3181bac4b2

10. Laufer I, lorgulescu JB, Chapman T, Lis E, Shi W, Zhang Z et al. Local disease control for spinal metastases following "separation surgery" and adjuvant hypofractionated or high-dose single-fraction stereotactic radiosurgery: outcome analysis in 186 patients. J Neurosurg Spine. 2013;18(3):207-14. doi:10.3171/2012.11.SPINE12111

11. Maranzano E, Latini P. Effectiveness of radiation therapy without surgery in metastatic spinal cord compression: final results from a prospective trial. Int J Radiat Oncol Biol Phys. 1995;32(4):959-67. doi:10.1016/0360-3016(95)00572-G

12. Yamada Y, Lovelock DM, Yenice KM, Bilsky MH, Hunt MA, Zatcky $J$ et al. Multifractionated image-guided and stereotactic intensity-modulated radiotherapy of paraspinal tumors: a preliminary report. Int J Radiat Oncol Biol Phys. 2005;62(1):53-61. doi:10.1016/j.ijrobp.2004.09.006

13. Gerszten PC, Mendel E, Yamada Y. Radiotherapy and radiosurgery for metastatic spine disease: what are the options, indications, and outcomes? Spine (Phila Pa 1976). 2009;34(22 Suppl):S78 -92. doi:10.1097/BRS.0b013e3181b8b6f5

14. Garg AK, Shiu AS, Yang J, Wang XS, Allen P, Brown BW et al. Phase 1/2 trial of single-session stereotactic body radiotherapy for previously unirradiated spinal metastases. Cancer. 2012;118(20):5069-77. doi:10.1002/cncr.27530

15. Joaquim AF, Ghizoni E, Tedeschi H, Pereira EB, Giacomini LA. Stereotactic radiosurgery for spinal metastases: a literature review. Einstein (São Paulo). 2013;11(2):247-55. doi:10.1590/S1679-45082013000200020

16. Tokuhashi Y, Matsuzaki H, Oda H, Oshima M, Ryu J. A revised scoring system for preoperative evaluation of metastatic spine tumor prognosis. Spine (Phila Pa 1976). 2005;30(19):2186-91. doi:10.1097/01.brs.0000180401.06919.a5 
17. Tomita K, Kawahara N, Kobayashi T, Yoshida A, Murakami H, Akamaru T. et al. Surgical strategy for spinal metastases. Spine (Phila Pa 1976). 2001;26(3):298-306. doi:10.1097/00007632-200102010-00016

18. Yamashita T, Siemionow KB, Mroz TE, Podichetty V, Lieberman IH. A prospective analysis of prognostic factors in patients with spinal metastases: use of the revised Tokuhashi score. Spine (Phila Pa 1976). 2011;36(11):910-7. doi:10.1097/BRS.0b013e3181e56ec1

19. Bilsky MH, Laufer I, Fourney DR, Groff M, Schmidt MH, Varga PP et al. Reliability analysis of the epidural spinal cord compression scale.J Neurosurg Spine. 2010;13(3):324-8. doi:10.3171/2010.3.SPINE09459

20. Fisher CG, DiPaola CP, Ryken TC, Bilsky MH, Shaffrey Cl, Berven SH et al. A novel classification system for spinal instability in neoplastic disease: an evidence-based approach and expert consensus from the Spine Oncology Study Group. Spine (Phila Pa 1976). 2010;35(22):E1221-9. doi:10.1097/BRS.0b013e3181e16ae2

21. Heng DY, Signorovitch J, Swallow E, Li N, Zhong Y, Qin P et al. Comparative effectiveness of second-line targeted therapies for metastatic renal cell carcinoma: a systematic review and meta-analysis of real-world observational studies. PLoS One. 2014;9(12):e114264. doi:10.1371/journal.pone.0114264

22. Rades D, Karstens JH, Hoskin PJ, Rudat V, Veninga T, Schild SE et al. Escalation of radiation dose beyond 30 Gy in 10 fractions for metastatic spinal cord compression. Int J Radiat Oncol Biol Phys. 2007;67(2):525-31. doi:10.1016/j.ijrobp.2006.09.025

23. Garcia-Barros M, Paris F, Cordon-Cardo C, Lyden D, Rafii S, Haimovitz-Friedman A et al. Tumor response to radiotherapy regulated by endothelial cell apoptosis. Science. 2003;300(5622):1155-9. doi:10.1126/science.1082504

24. Moulding HD, Elder JB, Lis E, Lovelock DM, Zhang Z, Yamada Y et al. Local disease control after decompressive surgery and adjuvant high-dose single-fraction radiosurgery for spine metastases.J Neurosurg Spine. 2010;13(1):87-93. doi:10.3171/2010.3.SPINE09639

25. Katagiri $H$, Takahashi M, Inagaki J, Kobayashi $H$, Sugiura $H$, Yamamura $S$ et al. Clinical results of nonsurgical treatment for spinal metastases. Int J Radiat Oncol Biol Phys. 1998;42(5):1127-32. doi:10.1016/S0360-3016(98)00288-0

26. Gerszten PC, Burton SA, Ozhasoglu C, Welch WC. Radiosurgery for spinal metastases: clinical experience in 500 cases from a single institution. Spine (Phila Pa 1976). 2007;32(2):193-9. doi:10.1097/01.brs.0000251863.76595.a2

27. Gilbert RW, Kim JH, Posner JB. Epidural spinal cord compression from metastatic tumor: diagnosis and treatment. Ann Neurol. 1978;3(1):40-51. doi:10.1002/ana.410030107

28. Rades D, Fehlauer F, Schulte R, Veninga T, Stalpers LJ, Basic $\mathrm{H}$ et al. Prognostic factors for local control and survival after radiotherapy of metastatic spinal cord compression. J Clin Oncol. 2006;24(21):3388-93. doi:10.1200/JC0.2005.05.0542

29. Rades D, Fehlauer F, Stalpers LJ, Wildfang I, Zschenker O, Schild SE et al. A prospective evaluation of two radiotherapy schedules with 10 versus 20 fractions for the treatment of metastatic spinal cord compression: final results of a multicenter study. Cancer. 2004;101(11):2687-92. doi:10.1002/cncr.20633

30. Guckenberger M, Mantel F, Gerszten PC, Flickinger JC, Sahgal A, Létourneau D et al. Safety and efficacy of stereotactic body radiotherapy as primary treatment for vertebral metastases: a multi-institutional analysis. Radiat Oncol. 2014;9(1):226. doi:10.1186/s13014-014-0226-2

31. Patchell RA, Tibbs PA, Regine WF, Payne R, Saris S, Kryscio RJ et al. Direct decompressive surgical resection in the treatment of spinal cord compression caused by metastatic cancer: a randomised trial. Lancet. 2005;366(9486):643-8. doi:10.1016/S0140-6736(05)66954-1

32. Mazura JC, Karimi S, Pauliah M, Banihashemi MA, Gobin YP, Bilsky MH et al. Dynamic contrast-enhanced magnetic resonance perfusion compared with digital subtraction angiography for the evaluation of extradural spinal metastases: a pilot study. Spine (Phila Pa 1976). 2014;39(16):E950-4. doi:10.1097/BRS.0000000000000409

33. Laufer I, Lis E, Pisinski L, Akhurst T, Bilsky MH. The accuracy of [(18)F]fluorodeoxyglucose positron emission tomography as confirmed by biopsy in the diagnosis of spine metastases in a cancer population. Neurosurgery. 2009;64(1):107-13. doi:10.1227/01.NEU.0000335176.98788.A1

34. Quinn JA, DeAngelis LM. Neurologic emergencies in the cancer patient. Semin Oncol. 2000;27(3):311-21.

35. Mendel E, Bourekas E, Gerszten P, Golan JD. Percutaneous techniques in the treatment of spine tumors: what are the diagnostic and therapeutic indications and outcomes? Spine (Phila Pa 1976). 2009;34(22 Suppl):S93-100. doi:10.1097/BRS.0b013e3181b77895

36. Brown PD, Stafford SL, Schild SE, Martenson JA, Schiff D. Metastatic spinal cord compression in patients with colorectal cancer. J Neurooncol. 1999;44(2):175-80. doi:10.1023/A:1006312306713

37. Cloyd JM, Acosta FL Jr, Polley MY, Ames CP. En bloc resection for primary and metastatic tumors of the spine: a systematic review of the literature. Neurosurgery. 2010;67(2):435-44. doi:10.1227/01.NEU.0000371987.85090.FF

38. Berenson J, Pflugmacher R, Jarzem P, Zonder J, Schechtman K, Tillman JB et al. Balloon kyphoplasty versus non-surgical fracture management for treatment of painful vertebral body compression fractures in patients with cancer: a multicentre, randomised controlled trial. Lancet Oncol. 2011;12(3):225-35. doi:10.1016/S1470-2045(11)70008-0 